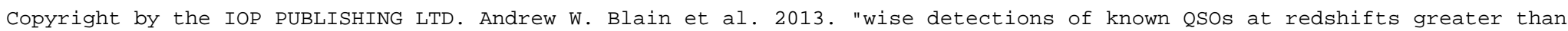
six," ApJ 778113 doi:10.1088/0004-637x/778/2/113

\title{
WISE DETECTIONS OF KNOWN QSOs AT REDSHIFTS GREATER THAN SIX
}

\author{
Andrew W. Blain ${ }^{1}$, Roberto Asser ${ }^{2,9,10}$, Daniel Stern ${ }^{2}$, Chao-Wei Tsai $^{2,9}$, Peter Eisenhardt $^{2}$, Carrie Bridge $^{3}$, \\ Dominic Benford ${ }^{4}$, Tom JarretT ${ }^{5}$, Roc Cutri ${ }^{6}$, Sara Petty ${ }^{7}$, Jingwen Wu ${ }^{8}$, and Edward L. Wright ${ }^{8}$ \\ ${ }^{1}$ Physics \& Astronomy, University of Leicester, 1 University Road, Leicester LE1 7RH, UK; ab520@ @ le.ac.uk \\ ${ }^{2}$ Jet Propulsion Laboratory, California Institute of Technology, Pasadena, CA 91109, USA \\ ${ }^{3}$ California Institute of Technology, 249-17, Pasadena, CA 91125, USA \\ ${ }^{4}$ Goddard Space Flight Center, Greenbelt, MD 20771, USA \\ ${ }^{5}$ Astronomy Department, University of Cape Town, South Africa \\ ${ }^{6}$ Infrared Processing and Analysis Center, California Institute of Technology, MS100-22, Pasadena, CA 91125, USA \\ ${ }^{7}$ Virginia Tech, Department of Physics MC 0435, 910 Drillfield Drive, Blacksburg, VA 24061, USA \\ ${ }^{8}$ Division of Astronomy \& Astrophysics, University of California, Los Angeles, Physics and Astronomy Building, \\ 430 Portola Plaza, Los Angeles, CA 90095-1547, USA \\ Received 2012 August 15; accepted 2013 September 25; published 2013 November 11
}

\begin{abstract}
We present WISE All-Sky mid-infrared (IR) survey detections of 55\% (17/31) of the known QSOs at $z>6$ from a range of surveys: the SDSS, the CFHT-LS, FIRST, Spitzer, and UKIDSS. The WISE catalog thus provides a substantial increase in the quantity of IR data available for these sources: 17 are detected in the WISE W1 (3.4 $\mu \mathrm{m})$ band, 16 in W2 $(4.6 \mu \mathrm{m}), 3$ in W3 $(12 \mu \mathrm{m})$, and 0 in W4 $(22 \mu \mathrm{m})$. This is particularly important with Spitzer in its warm-mission phase and no faint follow-up capability at wavelengths longward of $5 \mu \mathrm{m}$ until the launch of James Webb Space Telescope (JWST). WISE thus provides a useful tool for understanding QSOs found in forthcoming large-area optical/IR sky surveys using PanSTARRS, SkyMapper, VISTA, DES, and LSST. The rest-UV properties of the WISE-detected and the WISE-non-detected samples differ: the detections have brighter $i / z$-band magnitudes and redder rest-UV colors. This suggests that a more aggressive hunt for very high redshift QSOs by combining WISE W1 and W2 data with red, observed optical colors could be effective at least for a subset of dusty candidate QSOs. Stacking the WISE images of the WISE-non-detected QSOs indicates that they are, on average, significantly fainter than the WISE-detected examples, and are thus not narrowly missing detection in the WISE catalog. The WISE catalog detection of three of our sample in the W3 band indicates that their mid-IR flux can be detected individually, although there is no stacked W3 detection of sources detected in W1 but not W3. Stacking analyses of WISE data for large active galactic nucleus samples will be a useful tool, and high-redshift QSOs of all types will be easy targets for JWST.
\end{abstract}

Key words: galaxies: active - galaxies: evolution - infrared: galaxies - quasars: general

Online-only material: color figure

\section{INTRODUCTION}

At the highest redshifts, QSOs provide excellent tools for probing the process and end of reionization and provide signposts to some of the most active locations and phases in the process of galaxy evolution. A variety of surveys has been used to select active galaxies at high redshifts on the grounds of their optical and near-infrared (IR) colors. The optical Sloan Digital Sky Survey (SDSS; Abazajian et al. 2009) has mapped onequarter of the sky, from which the high-redshift QSOs have been selected and studied by Fan et al. (2001, 2003, 2004, 2006) and Jiang et al. (2008). A deeper, narrower optical-IR QSO survey has also been conducted by the Canada-France-Hawaii Telescope (CFHT) Legacy Survey (e.g., Willott et al. 2007, 2010). Combining near-IR imaging data in the selection criteria, the UKIDSS survey currently holds the QSO redshift record at $z=7.086$ (Mortlock et al. 2011). The southern $2 \mathrm{dF}$ and $6 \mathrm{dF}$ surveys (Croom et al. 2004; Heath Jones et al. 2009), based on digitized optical plate imaging and the IR Two Micron All-Sky Survey (2MASS), respectively, provide a substantial increase in the area surveyed for color-selected spectroscopically confirmed QSOs beyond the footprint of the SDSS. One further

\footnotetext{
9 NASA Postdoctoral Program (NPP).

${ }^{10}$ Also at Division of Astronomy, and Astrophysics, University of California, Los Angeles, Physics and Astronomy Building, 430 Portola Plaza, Los Angeles, CA 90095-1547, USA.
}

optically faint QSO was independently identified in the $8.5 \mathrm{deg}^{2}$ Spitzer Deep, Wide-Field Survey in Boötes by McGreer et al. (2006) and Stern et al. (2007). A current summary of references to $z>6$ QSOs can be found in Wang et al. (2011b). Deep observations using Spitzer have confirmed that at least some of the highest-redshift QSOs are detectable in the mid-IR (Charmandaris et al. 2004; Hines et al. 2006; Jiang et al. 2006; Stern et al. 2007), with properties not dramatically different than lower-redshift examples (Elvis et al. 1994; Staguhn et al. 2005). Some high-redshift QSOs are certainly also very rich in gas and dust, and provide most of the significant detections of ultraluminous galaxies in molecular lines at the highest redshifts (Wang et al. 2011a; Maiolino et al. 2012). There appears to be significant variation in the amounts of both the hottest and the total dust present, however (see Jiang et al. 2010). Some relevant results of analyzing spectral energy distributions (SEDs) of high-redshift active galaxies based on wideband UV-radio observations, including data from Spitzer and Herschel, are presented in Leipski \& Meisenheimer (2012); in particular, the long-wavelength far-IR and submillimeter properties of high-redshift active galaxies are discussed by Leipski et al. (2013).

The recent spectroscopic confirmation by Mortlock et al. (2011) of the first bright near-IR-selected QSO at $z>7$ spurred a search for the properties of known QSOs at high redshifts in the WISE All-Sky survey (Wright et al. 2010). WISE has mapped the whole sky in four IR bands, centered at 3.4, 4.6, 
12 , and $22 \mu \mathrm{m}$ (bands $\mathrm{W} 1-\mathrm{W} 4$, respectively), with the most relevant bands $\mathrm{W} 1$ and $\mathrm{W} 2$ reaching a typical $5 \sigma$ sensitivity of 6.8 and $9.8 \mu \mathrm{Jy}$, respectively, and an angular resolution of about 6 arcseconds. As a result of the polar orbit of WISE, depths vary across the sky - there is deeper coverage at the poles than at the equator.

We compiled a list of all 31 published spectroscopically confirmed QSOs at $z>6$ and searched the WISE All-Sky data release products catalog (2012 March 14; Cutri et al. $2012)^{11}$ to find detections and limits of these objects in the WISE bands from 3.4 to $22 \mu \mathrm{m}$. The $\simeq 6$ arcseconds spatial resolution of WISE is approximately three times coarser than the 1.7 arcseconds available from Spitzer-IRAC at $3.6 \mu \mathrm{m}$, and the integration time per point on the sky from WISE is typically only two minutes. However, the images have excellent astrometry and a search of the WISE database provides sensitive mid-IR $(>5 \mu \mathrm{m})$ all-sky imaging, reaching many times deeper than existing surveys from IRAS and Akari. Seven of our targets have archival detections by Spitzer (Jiang et al. 2006; Stern et al. 2007), but most were published after the cryogenic phase of Spitzer was completed, and so WISE provides the only source of mid-IR information longward of $5 \mu \mathrm{m}$ on most of these targets until the launch of James Webb Space Telescope (JWST). Most of the detections are made in WISE bands W1 and W2, which approximately match the IRAC [3.6] and [4.5] bands that are still operating in the Spitzer warm mission. Table 1 presents 17 WISE detections, 14 in the main all-sky release source catalog with $>5 \sigma$ detections and 3 from the "Reject Table" 12 that contains $>3.5 \sigma$ detections. This confirms that Spitzer can easily continue to detect the highest-redshift QSOs while the warm mission continues.

High-redshift QSOs are typically selected on the grounds of their smooth, broad, power-law spectra redward of a strong redshifted Lyman-break feature that at these redshifts lies between the $i$ and $z$ bands for $z \sim 6$ and between the $z$ and $J$ bands for the $z=7.02$ UKIDSS QSO. The WISE bands alone provide little additional power to select these objects, owing to the relatively featureless continuum emission of active galactic nuclei (AGN) yielding similar colors across the WISE bands at all redshifts (see Figure 1). However, when WISE detects highredshift AGN, the measured fluxes provide a valuable measure of the rest-frame optical spectral energy distribution in the W1 and W2 bands and signs of hot dust emission from the immediate environment of the AGN can be studied using the W3- and W4-band data.

In Section 2, we discuss the WISE properties of the $z>6$ QSOs, and their multiwavelength properties in Section 3. The quoted magnitudes are in their native system (Vega for WISE, $\mathrm{AB}$ for SDSS) unless otherwise stated (note Figures 5 and 6).

\section{WISE PROPERTIES OF QSOs IDENTIFIED AT $z>6$}

The list of $17 z>6$ QSOs that have cataloged WISE detections are presented in Table 1, along with their flux densities or limits from the WISE catalogs. Of the 14 all-sky release detections in $\mathrm{W} 1,13$ are also detected in the $\mathrm{W} 2$ band; a subset of three are also listed as detections in W3; none are detected in W4. All of the reported detections-which are distinguished in the table by not having a null (...) entry for a noise estimate-have a WISE pipeline signal-to-noise $(\mathrm{S} / \mathrm{N})$ ratio value (wXsnr) greater than 3.5 for band $X=1,2$, or 3 . The

\footnotetext{
11 http://wise2.ipac.caltech.edu/docs/release/allsky/expsup

$12 \mathrm{http}: / /$ wise2.ipac.caltech.edu/docs/release/allsky/expsup/sec2_4a.html
}

WISE colors of these high-redshift QSOs are compared with a match of the WISE and the SDSS QSO catalogs (Schneider et al. 2007) in Figure 1. That match yielded WISE colors for 19,876 QSOs at $z<5.6$.

The WISE-detected high-redshift QSOs are all found at similar positions in the WISE W1-W2:W2-W3 color space, close to $(3.0,1.0)$, deep within the densest region of WISE colors for lower-redshift AGN (Figure 1). This highlights the difficulty of using WISE photometry alone to identify candidate highredshift QSOs. Where there are only WISE limits, the regions where QSOs may lie covers almost the whole of this color space. Combining WISE and optical/near-IR data to highlight candidates is more promising, as we discuss in Section 3.

The WISE depth of coverage varies across the sky, with depth increasing away from the ecliptic plane toward the poles. The detectability of a target QSO appears to be related to this factor at least in part. The number of individual imaging frames taken by WISE that were included in the creation of the WISE Atlas Images for the 14 detections in the All-Sky Release source catalog ranged from 11-48, with a median of 21 and an interquartile range of 13-28. These numbers are unchanged when the three Reject-Table QSOs are included. For the 14 WISE non-detections, the number of individual WISE $8 \mathrm{~s}$ exposures that contributed to the catalog entry was $12-24$, with a median of 15 and an interquartile range of 13-22. We discuss the results of stacking a total of 188 of these $8 \mathrm{~s}$ single-exposure WISE image frames for the 10 non-detected sources below and conclude that, based on their different WISE fluxes, this coverage effect alone is unlikely to account for the difference between the detection and non-detection of high-redshift QSOs by WISE.

The WISE Atlas Images corresponding to the high-redshift QSOs were inspected to ensure that the reported source was not affected by image artifacts or other problems. ULAS J1120+0641 is within 15 arcseconds of a bright star; however, the photometry is uncontaminated. The images for the undetected $z>6$ QSOs were also inspected, and while in some cases, as expected, net positive signals are found at the QSO position, they are all consistent with a non-detection and thus are correctly reported as non-detections in the pipeline-processed WISE catalog. Further data was taken in the W1 and W2 bands after the cryogen was exhausted, the "WISE Post-Cryo Survey Phase" of the mission. These data will be combined with the cryogenic mission data in the near future, resulting in an "AllWISE" catalog with an anticipated noise level about 1.4 times deeper over about $70 \%$ of the sky, increasing the utility of WISE for detecting QSOs samples further.

Five QSOs-SDSS J1030+0524, SDSS J1148+5251, QSO $\mathrm{J} 1250+3130$, QSO B1425+3326, and SDSS J1602+4228-have bright W1 and W2 detections. Their WISE images, along with the images of the highest-redshift target ULAS J1120+0641, are shown in Figure 2. ${ }^{13}$ In the case of SDSS 1030+0524, SDSS J1148+5251, and QSO B1425+3326, there are Spitzer measurements (Jiang et al. 2006; Stern et al. 2007) that are consistent with the WISE W1 and W2 flux densities reported in Table 1 . Three have a relatively large number of detections, whereas QSO J1250+3130 is a very typical source.

In order to investigate the non-detections further, both for the $\mathrm{W} 1$ and $\mathrm{W} 2$ bands in the non-detected targets and for the W3 and W4 bands in the detected targets, we stacked the WISE images

\footnotetext{
13 We also compare the observed-frame optical-mid-IR SEDs for four of these objects in Figure 4. We exclude QSO B1425+3326 owing to concerns about a contaminating source, although its $i$ band to WISE colors are not unusual compared with the other detections.
} 

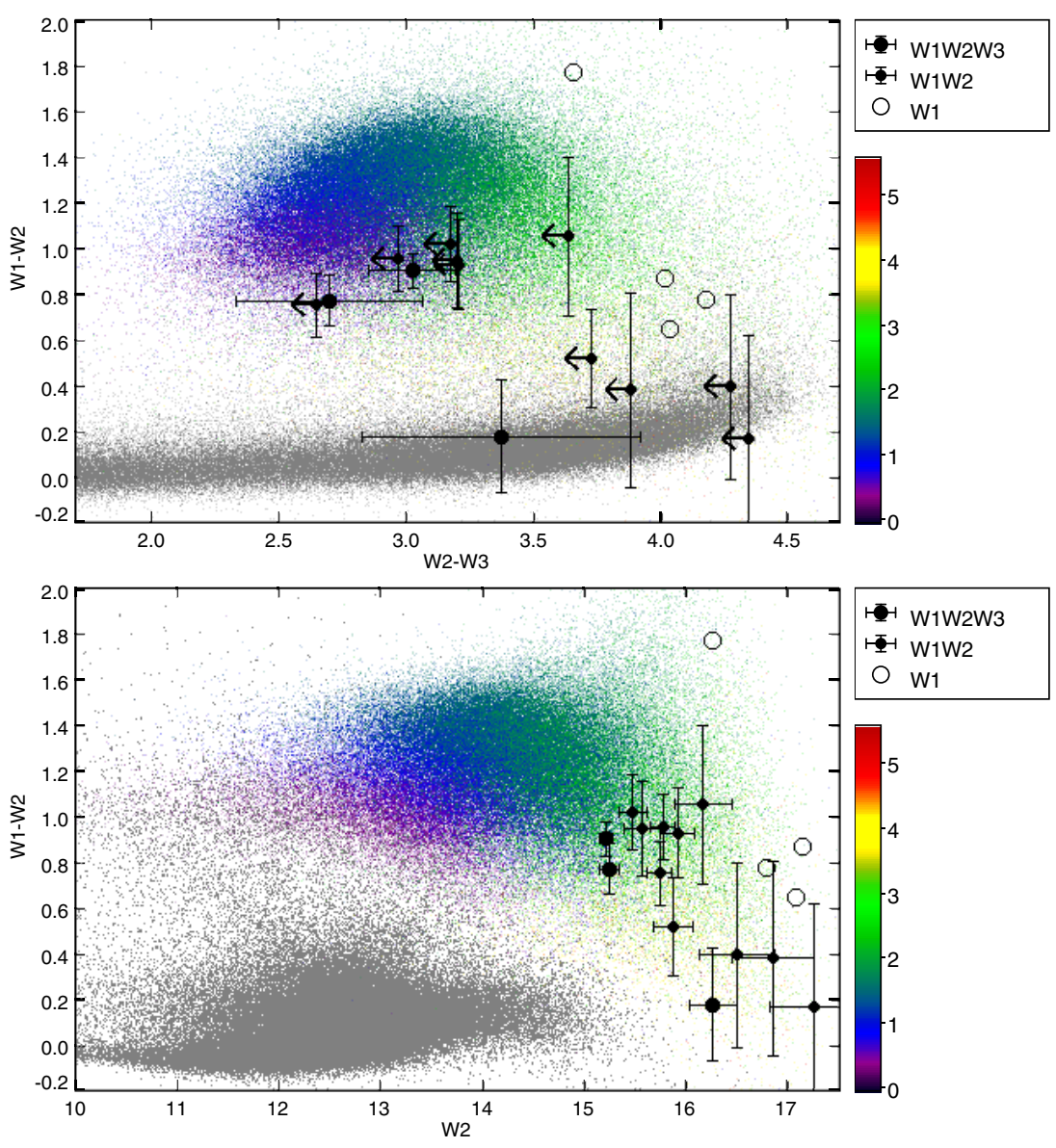

Figure 1. WISE color-color and color-magnitude diagrams for $z>6$ QSOs (filled black circles) and a large sample of QSOs matched between SDSS and WISE (colored points) that are detected in all three relevant WISE bands. Large solid circles represent $z>6$ QSOs with detections in all three of the bands W1/W2/W3. Smaller solid circles represent two-band detections in W1/W2. Open circles represent detections in W1 alone and are included for completeness only. Small filled circles with limit arrows represent the W2-W3 color if the W3 flux is at the limit listed in Table 1. The mid-IR properties of the SDSS QSO sample at $z<5.4$ (colored field; Schneider et al. 2007) were obtained from a cross match with the WISE All-sky Data Release catalog. Cataloged WISE objects within 3.5 arcseconds of the reported SDSS QSO survey positions were assumed to be correct identifications. The gray (lower) cloud of points represents a sample of low-redshift galaxies (see Wright et al. 2010; Jarrett et al. 2011) to illustrate the underlying galaxy populations.

(A color version of this figure is available in the online journal.)

from the QSO positions. In order to overcome the variations in the coverage of different targets, the whole ensemble of $8 \mathrm{~s}$ duration single-exposure WISE images that overlapped the target positions were extracted from the WISE Image Atlas and stacked with hot pixels and bright sources masked in order to probe the net flux density of the sample. This was done for all the targets without nearby bright objects, corresponding to $300 \mathrm{~W} 1$ frames, $300 \mathrm{~W} 2$ frames, $297 \mathrm{~W} 3$ frames, and 297 W4 frames for 16 (of 17 in total) high-redshift QSOs with detections in the bluer WISE bands. Stacking the frames for the 10 sources uncontaminated by nearby brighter objects (of 14 in total) without WISE detections in $\mathrm{W} 1$ or $\mathrm{W} 2$ provided a total of 188 frames in each of these bands. These stacked images are shown in Figure 3, with the same grayscale display range as shown in Figure 2. Note that the pixel scale in the W4 images is twice that in the other three bands. There is reasonably clear detection of the WISE-cataloged objects in the stack in both the $\mathrm{W} 1$ and $\mathrm{W} 2$ bands at $\mathrm{S} / \mathrm{N}$ ratios of 13.5 and 10.9, respectively. By comparison, the stack of the non-detected QSOs shows no sign of emission in the W1 or W2 bands (Figure 3), with a noise level in the stacked image that is $26 \%$ greater, reflecting the lesser number of uncontaminated fields included in the stack of non-detected targets (188 versus 300 ). There are no indications of any net detections in the longer WISE bands, W3 and W4, for any subsamples, which is reasonable given the sensitivity and the modest number of stacked fields.

Taken at face-value, this indicates that the fraction of Type-1 QSOs at $z>6$ that are not detected in the WISE catalog are systematically and substantially fainter in WISE bands W1 and W2 than the WISE-detected QSOs; they are not just a little fainter with the non-detections just evading detection below a formal flux limit. We now estimate the relative brightness of the WISE-detected and WISE-non-detected QSOs. We combine the results in bands W1 and W2 based on the results of the stacks described above. The average S/N ratio of the WISE-detected QSOs is 12.2 (the mean of 13.5 and 10.9) and the relative noise level in the stack of the non-WISE-detected QSOs is 1.26. Taking a $3 \sigma$ limit for the WISE non-detected sources, this gives a flux ratio of $12.2 /(3 \times 1.26) \simeq 3.2$. We interpret this as meaning that the WISE-non-detected QSOs in the sample considered here are likely to be, on average, a factor of about three times fainter than the WISE-detected QSOs.

The number of galaxies in this stack is very modest, however, and so the statistical power is limited. Nevertheless, there does appear to be a real difference in the mid-IR flux densities of the WISE-detected and WISE-non-detected $z>6$ QSO targets by an amount comparable to the offsets in their rest-UV optical magnitudes discussed further in Section 3. 


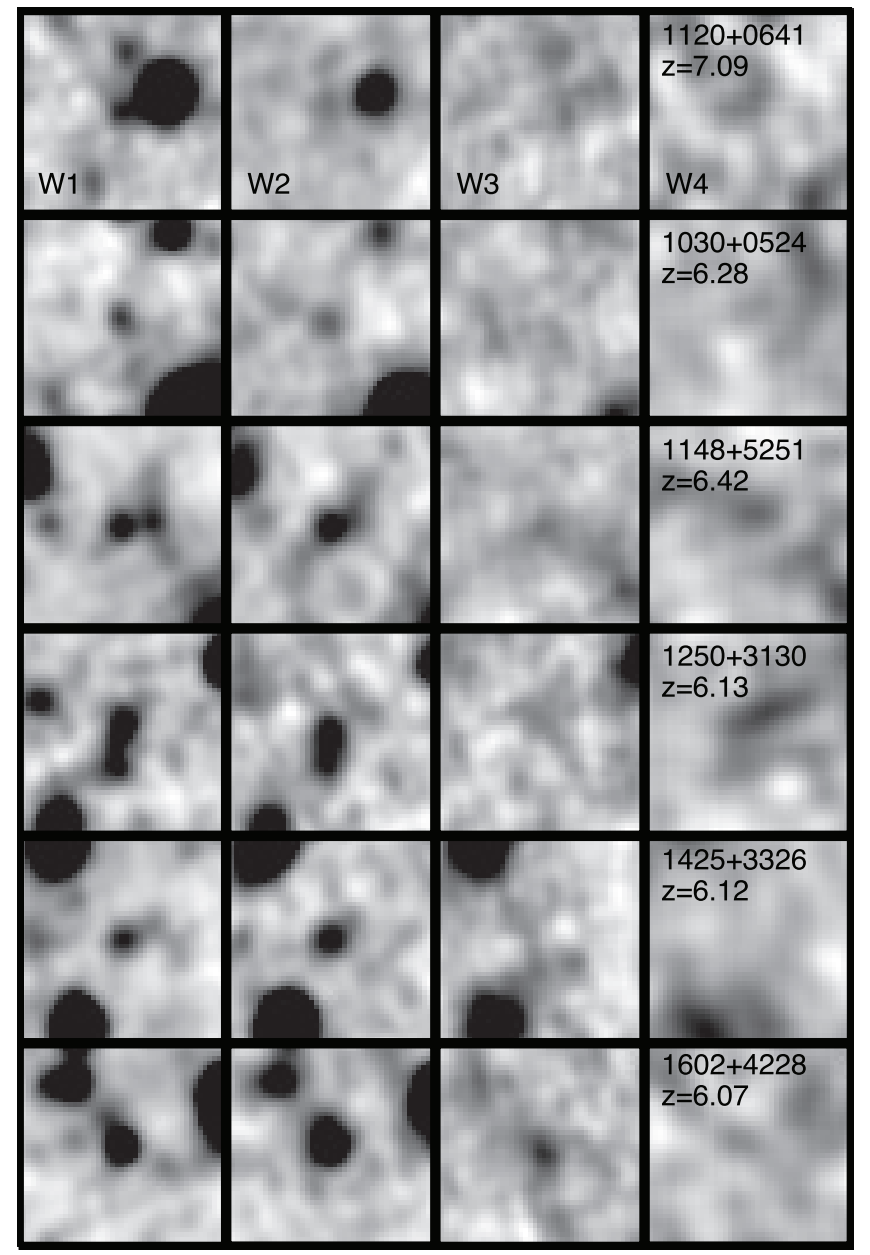

Figure 2. Postage stamp images, 60 arcseconds on a side, centered on the QSO position in WISE bands W1, W2, W3, W4 (from left to right along each row). From top to bottom, we show a different QSO in each row (labeled in the last column with their redshifts): ULAS J1120+0641, SDSS J1030+0524, SDSS $\mathrm{J} 1148+5251$, QSO J1250+3130, QSO B1425+3326/SDWFS J1427+3312, and SDSS J1602+4228. QSO J1250+3130 has a nearby companion, but far enough away from the WISE counterpart to ensure that the photometry remains accurate. The grayscale intensity is chosen to give an effective representation of the noise and so bright sources are saturated black.

\section{DISCUSSION}

We now discuss the WISE properties of the $z>6$ QSOs in the context of their optical and near-IR emission. The components of the emission that contributes to the brighter WISE-detected examples are investigated using SED models in the WISE bands in Figure 4. They are not significantly different from those previously reported at low and modest redshifts for Spitzer-

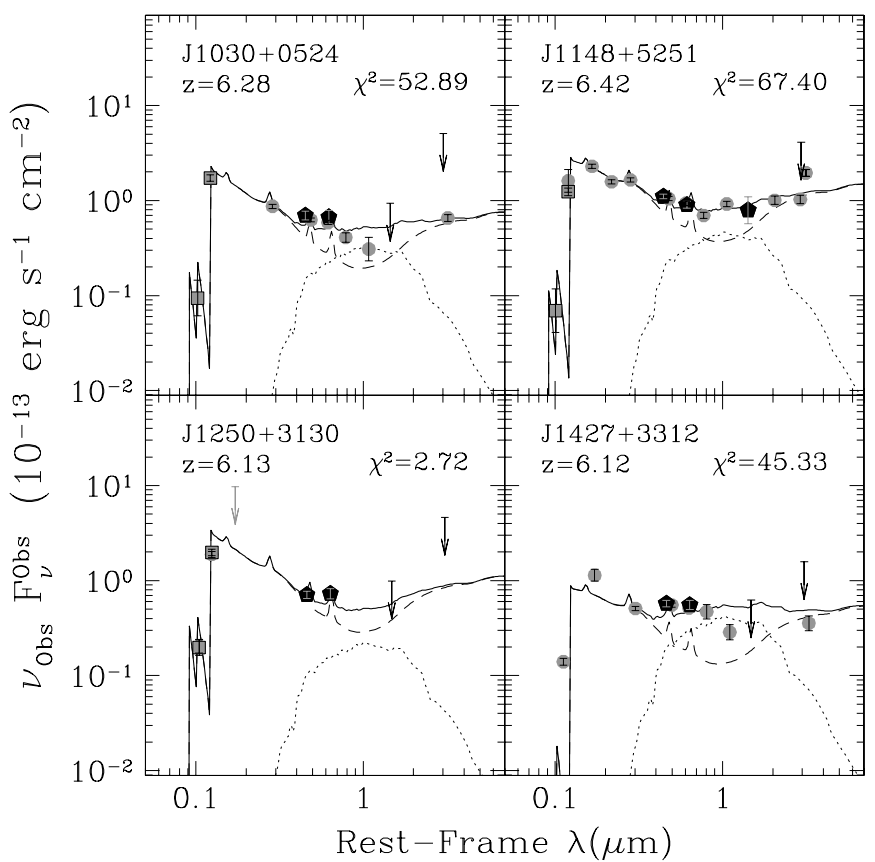

Figure 4. SEDs of four secure WISE 2/3 band detections from the $z>6$ QSO sample. A combination of different SED component templates (Assef et al. 2010), which were developed for data in the NDWFS Böotes field and are adequate to describe the WISE data, are also shown for an AGN (solid line) and an evolved stellar population (dotted line). The addition of other template components, for example, for a star-forming galaxy (Sbc/Im) component, does not improve the fits. The WISE data is highlighted by filled black pentagons and upper limits at the longer wavelengths. SDSS data is represented by gray squares and Spitzer and other sources by gray circles. J1250+3130 provides a good example of how data from WISE can provide a valuable insight into the relative strengths of different SED components from a typical WISE-detected example without Spitzer data. The sources of the data are referenced in Table 1. In all four cases, the WISE data and limits in W1 and W2 clearly limit the possible contribution from an evolved stellar population, while the information from W3 and W4 provides a valuable limit to the possible normalization and/or slope of the power-law mid-IR AGN component in the event that Spitzer data was not available, as is the case for $\mathrm{J} 1250+3130$. Clearly, the availability of Spitzer data provides much more information.

detected QSOs (Richards et al. 2006; Stern et al. 2012). Deep Spitzer observations of high-redshift QSOs (Jiang et al. 2010) have revealed some QSOs at $z \sim 6$ with little or no hot dust emission in the $24 \mu \mathrm{m}$ band. The larger number of much shallower observations by WISE in the W3 $(12 \mu \mathrm{m})$ band, which is sensitive to the hottest dust, could eventually be stacked to help to reveal whether these objects are unusual or typical. At face value, our $z>6$ sample indicates no obvious difference in the shapes of the SEDs of the WISE-detected and WISE-nondetected $z>6$ QSOs considered here to the depth of the survey.

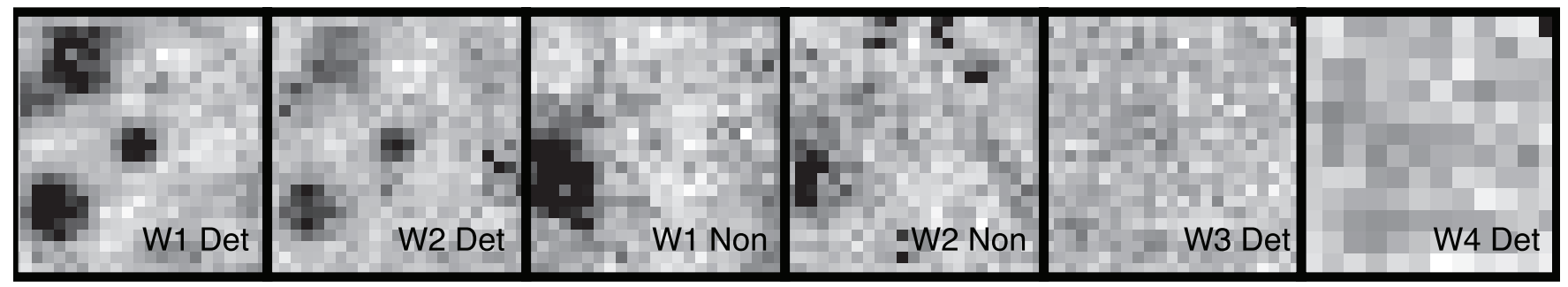

Figure 3. Results of stacking various combinations of the WISE images of $z>6$ QSOs. From left to right, a stack of the W1 and W2 images for $16 / 17$ of the W1-detected QSOs are shown as a comparison (W1 Det and W2 Det). Stacked W1 and W2 images for 10/14 of the non-detected objects with no nearby bright sources are then shown (W1 Non and W2 Non). Finally, W3 and W4 images are stacked from 13/14 W1-detected objects without W3 detections (W3 Det and W4 Det). Sources were excluded from the stacks on the grounds of a nearby brighter source that contributed light at the center of the stack; ULAS $1120+0641$ alone was excluded from the detected (Det) sources. The total number of $8 \mathrm{~s}$ duration WISE single-exposure image frames combined in these stacks is 300, 300, 188, 188, 297, and 297 respectively. 

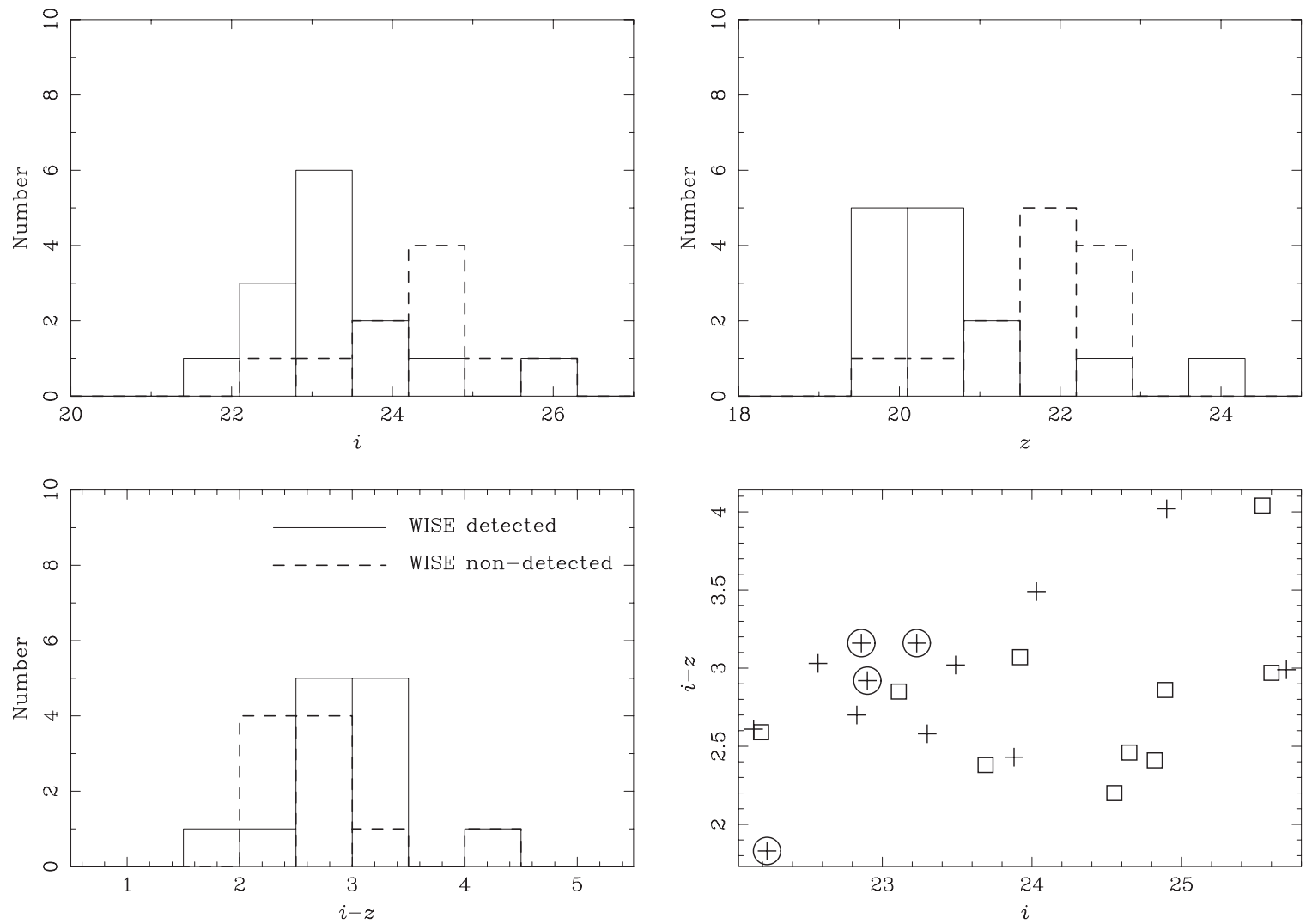

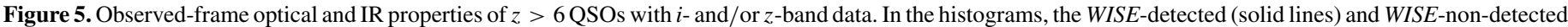

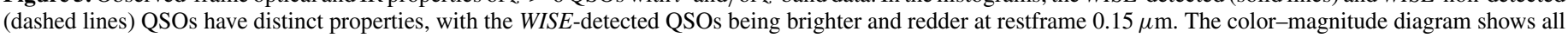

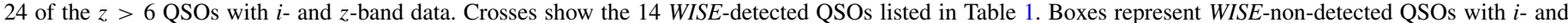

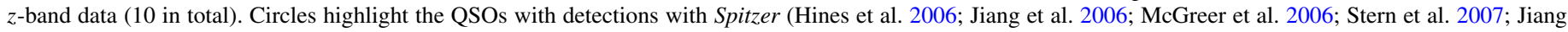
et al. 2010; see Table 1). An outlier, QSO J1630+4012, has been removed (Kim et al. 2009). All magnitudes are AB.

There seems to be little possibility that the WISE colors could be affected dramatically by redshifted line emission. In the range $6<z<7$, Ly $\alpha$ lies in the $i$ and $z$ bands, while $\mathrm{H} \alpha$ is in WISE $\mathrm{W} 2$ and $\mathrm{H} \beta$ is in WISE W1. The average restframe equivalent width is $194 \AA$ and $46 \AA$ for $\mathrm{H} \alpha$ and $\mathrm{H} \beta$ from the SDSS QSO template of vanden Berk et al. (2001). Redshifting these lines from $z=6.5$ would correspond to equivalent widths of $0.15 \mu \mathrm{m}$ for $\mathrm{H} \alpha$ in $\mathrm{W} 2$ and $0.035 \mu \mathrm{m}$ for $\mathrm{H} \beta$ in $\mathrm{W} 1$, respectively. The fractional bandwidths are approximately $25 \%$, and so about $15 \%$ of the W2 flux and 5\% of the W1 flux might be expected to be contributed by these lines. Although there is evidence that the equivalent width of $\mathrm{H} \alpha$ increases in galaxies at higher redshifts (Shim et al. 2011), this line contamination effect is unlikely to be significant here in light of the size of the measurement uncertainties.

The WISE colors of the $z>6$ detections are compared with the WISE colors of larger samples of lower-redshift QSOs in Figure 1. The WISE-SDSS cross-matched sample has much more complete coverage of low-redshift objects than other surveys. The SDSS sample contains relatively few lowerredshift QSOs around (4.2, 0.4), in the region of WISE color space where the $z>6$ QSOs are found (Figure 1); it includes a larger, more complete sample of QSOs with matches in the WISE catalog at $3<z<5$ and extends to a maximum redshift of $z=5.41$. At modest redshifts, $z<2-3$, the WISE colors provide a selection comparable to that described by Stern et al. (2005) for more moderate redshifts due to the relative differences between the colors of evolved stellar light and the AGN power law that dominates at longer wavelengths, as revealed by the $\mathrm{W} 1-\mathrm{W} 2$ color (see Stern et al. 2012; the discussion below and Figure 4). However, despite the confirmed ability of WISE to detect QSOs out to the end of the epoch of reionization, we emphasize that the colors of the WISE-detected objects do not stand out from the much larger numbers of AGN at more moderate redshifts (Figure 1), as their colors are determined by a broken power-law SED that does not change very strongly with increasing redshift. Thus the discovery of purely WISE-selected samples of highredshift AGN are unlikely; there is no unambiguous signature of a very high redshift QSO $(z>6)$ from the WISE data alone at the WISE depth. The W1-W2 colors of the $z>6$ QSOs are typically bluer than those at low redshifts, as expected, but they are difficult to distinguish from $z \simeq 0.5$ star-forming galaxies as many are bluer than the $\mathrm{W} 1-\mathrm{W} 2>0.8$ color cut imposed in WISE color-based AGN selection methods (see Stern et al. 2012; Mateos et al. 2012; Assef et al. 2013).

Furthermore, most of the W3 flux values are upper limits and so the WISE points without error bars can move to the left in the W2-W3 color space of Figure 1, corresponding to bluer mid-IR colors. However, the detected $z>6$ QSOs remain within the cloud of lower-redshift QSOs and luminous IR galaxies (Wright et al. 2010; Jarrett et al. 2011) in this color space, preventing any easy sifting of QSO candidates from the WISE data.

WISE data add significantly to the available information about high-redshift candidates, as shown in Figures 4-6. The SEDs of four of the brighter WISE detections, whose images are shown in Figure 2, are compared with templates from Assef et al. (2010) in Figure 4. These fits allow an estimate of the relative SED contributions of an evolved stellar component and an AGN 


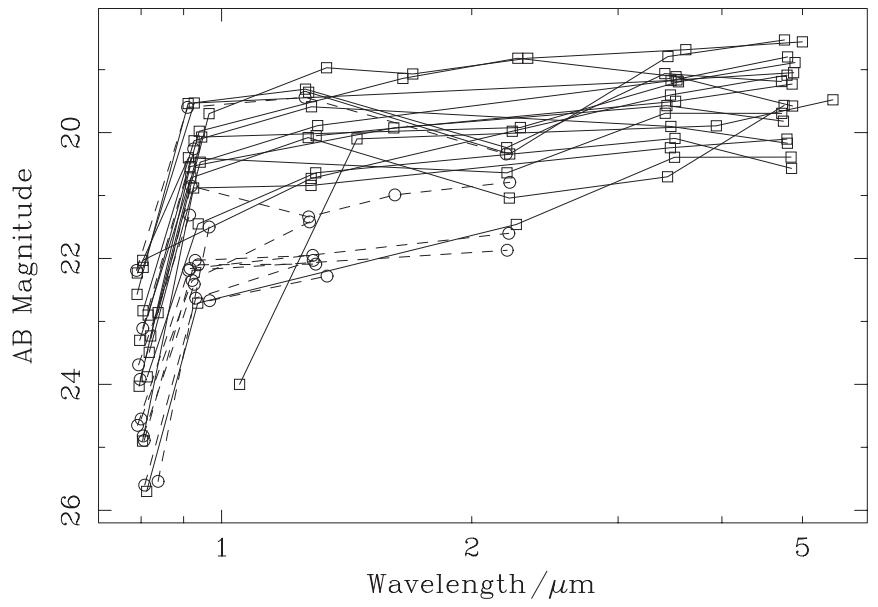

Figure 6. Observed-frame optical-mid-IR SEDs of $z>6$ QSOs from the $i$-band $(0.8 \mu \mathrm{m})$ to WISE W2 at $4.6 \mu \mathrm{m}$. The two rightmost clouds of points represent WISE bands W1 and W2. The relative wavelength of different points in the clouds represent the relative redshift for each QSO, offset from the wavelength of the band center at a precise redshift $z=6$. There are only three significant detections at longer wavelengths from WISE, so we plot the SEDs only to restframe $5 \mu \mathrm{m}$. WISE-detected QSOs have SEDs that extend longward of $3 \mu \mathrm{m}$ on the plot and are represented by square data points connected by solid lines, whereas WISE non-detected QSOs are represented by circular data points connected by dashed lines. Note that WISE-detected examples have typically slightly redder observed $i-z$ colors (see Figure 5). Note that at $z=6(7)$, Ly $\alpha$ is at $0.85(0.97) \mu \mathrm{m}$, potentially increasing the optical magnitudes, while $\mathrm{H} \alpha$ potentially enhances the W2 flux according to the template in vanden Berk et al. (2001), as shown by the Assef et al. (2010) template curves in Figure 4.

component. The AGN is described approximately by two power laws, with a break near $1 \mu \mathrm{m}$ (e.g., vanden Berk et al. 2001). These templates appear to provide a reasonable description of the SEDs of the $z>6$ QSOs. In no case is there compelling evidence that a large fraction of light from a young star-forming population $(\mathrm{Sbc} / \mathrm{Im})$ is required in the models to account for the data. Less precise SED information for more of the WISE-detected and non-detected QSOs is shown in Figure 6.

Seven of the 17 WISE-detected $z>6$ QSOs listed in Table 1 have Spitzer detections (see the footnotes to Table 1). They typically have a median of 3 bands of iz JHK photometry available, while the 14 WISE non-detected $z>6$ QSOs have a median of 2 detections in these bands.

The relative rest-UV colors of the WISE-detected and WISE-non-detected $z>6$ QSOs are compared in Figure 5. The relative magnitudes and colors, indicating that the WISE nondetections are both fainter by about two magnitudes in $i$ and $z$, have a modestly greater range in their $z$-band magnitudes, and are marginally bluer in $i-z$ by about half a magnitude. As discussed above, $i-z$ straddles the wavelength of Ly $\alpha$ at these redshifts and so this rest-UV color could be affected strongly by the column density of external and internal hydrogen absorption and the effects of extinction. Note that the highest-redshift example, ULAS J1120+0641, does not appear in this plot as Ly $\alpha$ has redshifted through both of the relevant bands at $z>7$. These differences are consistent with the WISE detectability amongst the $31 z>6$ QSOs being related to the fainter $z$-band emission from the targets not detected by WISE. If the rest-UV light comes from accretion (Figure 4) and is, on average, two magnitudes fainter in the WISE-non-detected sample than the WISE-detected one, then it could just reflect significantly different accretion powers between the subsamples without any major difference in the optical-mid-IR colors. Note that there is inadequate deep near-IR data (see also
Figure 6) to construct comparable histograms for the observed near-IR colors/magnitudes for these samples. Some information is available from deeper observations (e.g., Staguhn et al. 2005) and all the available information is included in the SEDs shown in Figure 6.

The large number of AGN candidates that are sure to be detected using PanSTARRS (Morganson et al. 2012), SkyMapper, VISTA, VST, DES, and LSST can be matched to the WISE catalog in order to identify particularly dusty examples, to get a better idea of their true luminosities, and, at the highest redshifts, to obtain more useful limits on their stellar masses. The significant detection rate of existing $z>6$ QSO samples in the WISE All-Sky Release catalog, mostly from within the SDSS footprint, and in the deeper, smaller area CFHT surveys offers a potentially large sample of future candidates. The WISE detection of a substantial fraction of known $z>6$ QSOs, and one at $z>7$, makes us optimistic that candidates at even higher redshifts could be found and isolated by taking advantage of a combination of deep wide-field optical-near-IR surveys and the WISE catalog. When WISE detects a QSO and a redshift is known, the WISE data also immediately provides limits to its hot dust emission from $\mathrm{W} 3$, to its rest-optical luminosity from $\mathrm{W} 1$ and W2, and even perhaps an evolved stellar population, all of which would otherwise be difficult to obtain until the launch of JWST.

\section{CONCLUSIONS}

We have searched for 31 known $z>6$ QSOs in the WISE All-Sky survey and detected 17/31 (55\%) in band W1, and 16,3 , and zero in bands $\mathrm{W} 2, \mathrm{~W} 3$, and $\mathrm{W} 4$, respectively. This significant detection rate for QSOs selected from both SDSS and deeper imaging should make WISE a useful resource for exploiting ongoing wide-optical/near-IR imaging surveys. The SED properties of the brighter WISE-detected objects are not unlike those of lower-redshift samples. From stacking the nondetected sample of 14 QSOs, their 3.4 and $4.6 \mu \mathrm{m}$ emission (in bands W1 and W2, respectively) appears to be significantly less powerful than that of the WISE-detected QSOs, indicating that there is likely to be a significant difference between the mid-IR luminosities of the two classes, with WISE-non-detection due to intrinsic faintness and not simply due to a matter of a chance appearance above the noise level in observations with low $\mathrm{S} / \mathrm{N}$ ratios.

We thank the anonymous referee for a careful reading and helpful suggestions. This publication makes use of data products from the Wide-field Infrared Survey Explorer (WISE). WISE is a joint project of the University of California, Los Angeles, and the Jet Propulsion Laboratory/California Institute of Technology, funded by the National Aeronautics and Space Administration. This research has made use of both the NASA/IPAC Infrared Science Archive (IRSA) and the NASA/IPAC Extragalactic Database (NED), which are operated by the Jet Propulsion Laboratory, California Institute of Technology, under contract with the National Aeronautics and Space Administration. R.J.A. was supported by an appointment to the NASA Postdoctoral Program at the Jet Propulsion Laboratory, administered by Oak Ridge Associated Universities through a contract with NASA.

Note added in proof. The enhanced AllWISE data release is scheduled for late 2013. The AllWISE catalogs are being compiled as this paper goes to press. Of the sources listed in Table 1, 16 are in the new source tables. The exception is SDSS 
Table 1

WISE Vega Magnitudes for WISE-detected QSOs at $z>6$, where a WISE W1 S/N ratio is Reported to be Greater than 3.5

\begin{tabular}{|c|c|c|c|c|c|c|c|c|c|c|c|c|}
\hline Name & R.A. & Decl. & Reference & $z$ & W1 & $\sigma_{1}$ & W2 & $\sigma_{2}$ & W3 & $\sigma_{3}$ & W4 & $\sigma_{4}$ \\
\hline CFHQS J0050+3445 & $00: 50: 06.67$ & $+34: 45: 22.6$ & Willott et al. (2010) & 6.25 & 16.482 & 0.073 & 15.734 & 0.119 & 13.084 & $\cdots$ & 9.609 & $\cdots$ \\
\hline CFHQS J0227-0605 & $02: 27: 43.33$ & $-06: 05: 31.4$ & Willott et al. (2009) & 6.20 & 17.713 & 0.211 & 17.071 & $\ldots$ & 13.038 & $\cdots$ & 9.498 & $\ldots$ \\
\hline SDSS J0353+0104 ${ }^{\mathrm{a}}$ & $03: 53: 49.72$ & $+01: 04: 04.4$ & Jiang et al. (2008) & 6.049 & 16.886 & 0.147 & 16.497 & 0.375 & 12.225 & $\ldots$ & 8.560 & $\ldots$ \\
\hline SDSS J1030+0524 $4^{\mathrm{a}, \mathrm{e}}$ & $10: 30: 27.10$ & $+05: 24: 55.0$ & Fan et al. (2001) & 6.28 & 16.512 & 0.114 & 15.567 & 0.174 & 12.364 & $\ldots$ & 8.372 & $\ldots$ \\
\hline QSO J1048+4637 $7^{\mathrm{a}, \mathrm{b}, \mathrm{c}, \mathrm{e}}$ & $10: 48: 45.05$ & $+46: 37: 18.3$ & Fan et al. (2003) & 6.23 & 16.430 & 0.080 & 16.259 & 0.233 & 12.885 & 0.495 & 8.843 & $\ldots$ \\
\hline ULAS J1120+0641 & $11: 20: 01.48$ & $+06: 41: 24.3$ & Mortlock et al. (2011) & 7.085 & 17.208 & 0.205 & 16.160 & 0.281 & 12.523 & $\ldots$ & 8.597 & . \\
\hline QSO J1137+3549a & $11: 37: 17.73$ & $+35: 49: 56.9$ & Fan et al. (2006) & 6.01 & 16.379 & 0.092 & 15.868 & 0.193 & 12.144 & $\ldots$ & 8.765 & $\ldots$ \\
\hline SDSS J1148+5251 & $11: 48: 16.64$ & $+52: 51: 50.30$ & Fan et al. (2003) & 6.43 & 16.007 & 0.062 & 15.242 & 0.093 & 12.544 & 0.352 & 8.598 & $\ldots$ \\
\hline QSO J1250+3130 & $12: 50: 51.93$ & $+31: 30: 21.9$ & Fan et al. (2006) & 6.13 & 16.489 & 0.096 & 15.474 & 0.136 & 12.302 & $\ldots$ & 8.473 & $\ldots$ \\
\hline ULAS J1319+0950b & $13: 19: 11.29$ & $+09: 50: 51.4$ & Mortlock et al. (2009) & 6.127 & 17.222 & 0.145 & 16.848 & 0.400 & 12.966 & $\ldots$ & 9.030 & $\ldots$ \\
\hline QSO B1425+3326 $6^{\mathrm{a}, \mathrm{h}, \mathrm{j}}$ & $14: 27: 38.59$ & $+33: 12: 42.0$ & McGreer et al. (2006) & 6.12 & 16.720 & 0.084 & 15.770 & 0.117 & 12.801 & $\ldots$ & 9.604 & $\ldots$ \\
\hline CFHQS J1429+5447 & $14: 29: 52.17$ & $+54: 47: 17.7$ & Willott et al. (2010) & 6.21 & 17.405 & 0.141 & 17.246 & 0.435 & 12.903 & $\ldots$ & 9.564 & . \\
\hline QSO J1602+4228 a,e & $16: 02: 53.98$ & $+42: 28: 24.9$ & Fan et al. (2004) & 6.07 & 16.107 & 0.046 & 15.209 & 0.062 & 12.184 & 0.158 & 9.526 & $\ldots$ \\
\hline QSO J1623+3112 a,e,f & $16: 23: 31.81$ & $+31: 12: 00.50$ & Fan et al. (2004) & 6.22 & 16.839 & 0.110 & 15.914 & 0.166 & 12.706 & $\cdots$ & 9.268 & $\cdots$ \\
\hline QSO J1630+4012a,e,i & $16: 30: 33.90$ & $+40: 12: 09.60$ & Fan et al. (2003) & 6.05 & 18.000 & 0.273 & 17.138 & $\ldots$ & 13.122 & $\ldots$ & 9.447 & $\ldots$ \\
\hline SDSS J2054-0005 & $20: 54: 06.49$ & $-00: 05: 14.80$ & Jiang et al. (2008) & 6.062 & 18.017 & 0.337 & 16.25 & $\ldots$ & 12.595 & $\ldots$ & 8.727 & $\ldots$ \\
\hline SDSS J2315-0023 & $23: 15: 46.57$ & $-00: 23: 58.1$ & Jiang et al. (2008) & 6.117 & 17.559 & 0.283 & 16.785 & $\cdots$ & 12.606 & $\ldots$ & 8.624 & . \\
\hline
\end{tabular}

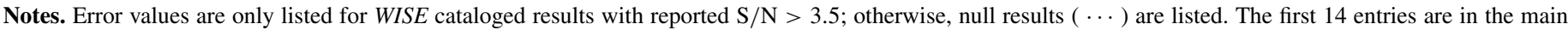

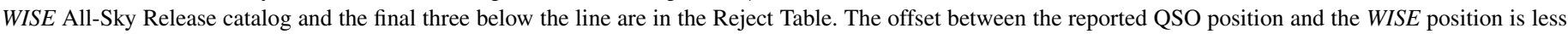

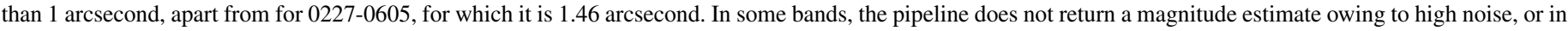

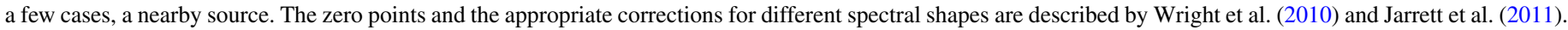
a These QSOs were observed but not detected in the millimeter-wave continuum (Wang et al. 2007, 2008a).

b These QSOs were detected in the millimeter-wave continuum (Wang et al. 2011b), implying a very large far-IR luminosity.

c $1048+4637$ was detected by at submillimeter wavelengths by Wang et al. (2008b), implying a very large far-IR luminosity.

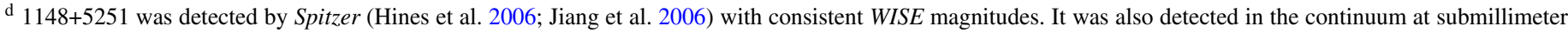

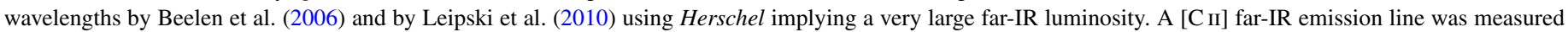
by Maiolino et al. (2012).

e These galaxies were detected by Spitzer (Jiang et al. 2006) with consistent magnitudes to WISE.

${ }^{f}$ An upper limit to a CO line flux was reported for this QSO by Wang et al. (2011a).

g Observed at 450 and $850 \mu \mathrm{m}$ by Robson et al. (2004). J1048+4637 was detected.

${ }^{\text {h }}$ Detected by Spitzer (Stern et al. 2007).

${ }^{\mathrm{i}}$ An upper limit to CO line flux was reported for this galaxy by Maiolino et al. (2007).

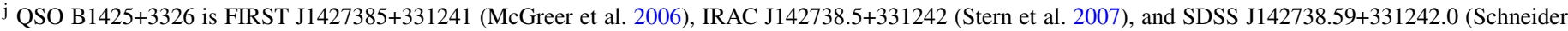
et al. 2007).

J2054-0005, which was listed in the All-sky Reject Table. Searching the 31 high-redshift targets in the new tables yields one additional target that satisfies the conditions for inclusion in the All-Sky Release Catalog and three additional targets that satisfy the conditions for being entered in the All-Sky Reject Table, while two sources are promoted from the Reject Table to the catalog and one source is demoted from the catalog to the Reject Table. Hence, the 14 targets listed in the All-Sky Release Catalog and the 3 in the Reject Table in Table 1 for the WISE All-Sky data release are likely to be changed to 16 and 4, respectively, in the AllWISE Catalog, giving an increased cataloged fraction of $65 \%$ for known QSOs at redshifts greater than six.

Facility: WISE

\section{REFERENCES}

Abazajian, K. N., Adelman-McCarthy, J. K., Agueros, M. A., et al. 2009, ApJS, 182,543

Assef, R. J., Kochanek, C. S., Brodwin, M., et al. 2010, ApJ, 713, 970

Assef, R. J., Stern, D., Kochanek, C. S., et al. 2013, ApJ, 772, 26

Beelen, A., Cox, P., Benford, D. J., et al. 2006, ApJ, 642, 108

Charmandaris, V., Uchida, K. I., Weedman, D., et al. 2004, ApJS, 154, 142

Croom, S. M., Smith, R. J., Boyle, B. J., et al. 2004, MNRAS, 349, 1397

Cutri, R. M., Wright, E. L., Conrow, T., et al. 2012, Explanatory Supplement to the WISE All-Sky Data Release Products, 1

Elvis, M., Wilkes, B., McDowell, J. C., et al. 1994, ApJS, 95, 1

Fan, X., Hennawi, J. F., Richards, G. T., et al. 2004, AJ, 128, 515
Fan, X., Narajanan, V. K., Lupton, R. H., et al. 2001, AJ, 122, 2833 Fan, X., Strauss, M. A., Richards, G. T., et al. 2006, AJ, 131, 1203 Fan, X., Strauss, M. A., Schneider, D. P., et al. 2003, AJ, 125, 1649 Heath Jones, D., Read, M. A., Saunders, W., et al. 2009, MNRAS, 399, 683 Hines, D. C., Krause, O., Rieke, G. H., et al. 2006, ApJL, 641, L85 Jarrett, T., Cohen, M., Masci, F., et al. 2011, ApJ, 735, 112 Jiang, L., Fan, X., Annis, J., et al. 2008, AJ, 135, 1057

Jiang, L., Fan, X., Brandt, W. N., et al. 2010, Natur, 464, 380 Jiang, L., Fan, X., Hines, D. C., et al. 2006, AJ, 132, 2127

Kim, S., Stiavelli, M., Trenti, M., et al. 2009, ApJ, 695, 809 Leipski, C., \& Meisenheimer, M. 2012, JPhCS, 372, 012037 Leipski, C., Meisenheimer, K., Klaas, U., et al. 2010, A\&A, 518, L34 Leipski, C., Meisenheimer, K., Walter, F., et al. 2013, ApJ, 772, 103 Maiolino, R., Gallerani, S., Neri, R., et al. 2012, MNRAS, 425, L66 Maiolino, R., Neri, R., Beelen, A., et al. 2007, A\&A, 472, L33

Mateos, S., Alonso-Herrero, A., Carrera, F. J., et al. 2012, MNRAS, 426, 3271 McGreer, I. D., Becker, R. H., Helfand, D. J., et al. 2006, ApJ, 652, 157

Morganson, E., De Rosa, G., Decarli, R., et al. 2012, AJ, 143, 142 Mortlock, D. J., Patel, M., Warren, S. J., et al. 2009, A\&A, 505, 97 Mortlock, D. J., Warren, S., Patel, M., et al. 2011, Natur, 474, 616

Richards, G. T., Lacy, M., Storrie-Lombardi, L. J., et al. 2006, ApJS, 166,470

Robson, I., Priddey, R. S., Isaak, K. G., \& McMahon, R. G. 2004, MNRAS, 351, L29

Schneider, D. P., Hall, P. B., Richards, G. T., et al. 2007, AJ, 134, 102

Shim, H., Chary, R.-R., Dickinson, M., et al. 2011, ApJ, 738, 69

Staguhn, J., Stern, D., Benford, D. J., et al. 2005, ApJ, 629, 633

Stern, D., Eisenhardt, T., Gorijian, V., et al. 2005, ApJ, 631, 163

Stern, D., Kirkpatrick, J. D., Allen, L. E., et al. 2007, ApJ, 663, 677

Stern, D. A., Assef, R. J., Benford, D. J., et al. 2012, ApJ, 753, 30 
vanden Berk, D., Richards, G. T., Bauer, A., et al. 2001, AJ, 122, 549

Wang, R., Carilli, C. L., Beelen, A., et al. 2007, AJ, 134, 617

Wang, R., Carilli, C. L., Wagg, J., et al. 2008a, ApJ, 687, 848

Wang, R., Wagg, J., Carilli, C. L., et al. 2008b, AJ, 135, 1201

Wang, R., Wagg, J., Carilli, C. L., et al. 2011a, ApJL, 739, L34
Wang, R., Wagg, J., Carilli, C. L., et al. 2011b, AJ, 142, 101

Willott, C., Delorme, P., Omont, A., et al. 2007, AJ, 134, 2435

Willott, C., Delorme, P., Reylé, C., et al. 2009, AJ, 137, 3541

Willott, C., Delorme, P., Reylé, C., et al. 2010, AJ, 139, 906

Wright, E. L., Eisenhardt, P., Mainzer, A., et al. 2010, AJ, 140, 1868 


\title{
ERRATUM: “WISE DETECTIONS OF KNOWN QSOs AT REDSHIFTS GREATER THAN SIX”
}

\author{
(2013, ApJ, 778, 113)
}

\author{
Andrew W. Blain ${ }^{1}$, Roberto Assef ${ }^{2,3,10}$, Daniel Stern $^{2}$, Chao-Wei Tsai $^{2,10}$, Peter Eisenhardt ${ }^{2}$, Carrie Bridge $^{4}$, \\ Dominic Benford ${ }^{5}$, Tom Jarrett ${ }^{6}$, Roc Cutri ${ }^{7}$, Sara Pettr ${ }^{8}$, Jingwen Wu ${ }^{9}$, And Edward L. Wright ${ }^{9}$ \\ ${ }^{1}$ Physics \& Astronomy, University of Leicester, 1 University Road, Leicester LE1 7RH, UK; ab520@ @e.ac.uk \\ 2 Jet Propulsion Laboratory, California Institute of Technology, Pasadena, CA 91109, USA \\ ${ }^{3}$ Núcleo de Astronomía de la Facultad de Ingeniería, Universidad Diego Portales, Av. Ejército 441, Santiago, Chile \\ ${ }^{4}$ California Institute of Technology, 249-17, Pasadena, CA 91125, USA \\ ${ }^{5}$ Goddard Space Flight Center, Greenbelt, MD 20771, USA \\ ${ }^{6}$ Astronomy Department, University of Cape Town, South Africa \\ ${ }^{7}$ Infrared Processing and Analysis Center, California Institute of Technology, MS100-22, Pasadena, CA 91125, USA \\ ${ }^{8}$ Virginia Tech, Department of Physics MC 0435, 910 Drillfield Drive, Blacksburg, VA 24061, USA \\ ${ }^{9}$ Division of Astronomy \& Astrophysics, University of California, Los Angeles, Physics and Astronomy Building, \\ 430 Portola Plaza, Los Angeles, CA 90095-1547, USA \\ Received 2014 January 6; published 2014 January 27
}

In the published version of this paper, Roberto Assef was mistakenly affiliated with the Division of Astronomy and Astrophysics at the University of California, Los Angeles. This is incorrect. Dr. Assef's affiliation correctly appears in this erratum as the Núcleo de Astronomía de la Facultad de Ingeniería, Universidad Diego Portales, Av. Ejército 441, Santiago, Chile.

IOP Publishing sincerely regrets this error.

\footnotetext{
${ }^{10}$ NASA Postdoctoral Program (NPP).
} 